\title{
2. Daten und Methoden des Deutschen Alterssurveys
}

\author{
Daniela Klaus \& Heribert Engstler
}

\section{Kernaussagen}

Der Deutsche Alterssurvey (DEAS) untersucht die Lebenssituationen und Alternsverläufe der Menschen in der zweiten Lebenshälfte über einen Zeitraum von bislang 18 Jahren: Zwischen 1996 und 2014 wurden fünf Erhebungen durchgeführt (1996, 2002, 2008, 2011, 2014). Befragt werden Personen ab ihrem 40. Lebensjahr.

Der DEAS kombiniert Quer- und Längsschnitterhebungen: Bisher haben 20.715 Personen an der Befragung teilgenommen. Sie haben insgesamt 33.410 mündliche Interviews gegeben. Bereits 6.623 Personen wurden zweimal oder öfter befragt.

Trotz einer rückläufigen Teilnahmebereitschaft weist der DEAS eine hohe Datenqualität auf: Die Teilnahmequote bei der Erstbefragung liegt zwischen 50,3 Prozent (1996) und 27,1 Prozent (2014). Dennoch bestehen nur geringe Selektivitäten hinsichtlich der Teilnahmewahrscheinlichkeit, die denen aus vergleichbaren Studien ähneln.

Die Studie deckt eine große Breite alter(n)srelevanter Themen ab: Informationen zu zentralen Lebensbereichen und Dimensionen von Lebensqualität werden über ein standardisiertes mündliches Interview sowie einen schriftlichen Fragebogen erhoben. Neben den Individualdaten stehen Kontextdaten auf Kreisebene und zu Merkmalen des Wohnumfelds zur Verfügung.

\subsection{Einleitung}

Der Deutsche Alterssurvey (DEAS) ist eine seit 1996 durchgeführte Befragung von Menschen in der zweiten Lebenshälfte in Deutschland. Mit der jüngsten DEAS-Erhebung im Jahr 2014 hat sich der Beobachtungszeitraum der Studie auf nunmehr 18 Jahre und fünf Erhebungen erweitert (1996, 2002, 2008, 2011, 2014). In Verbindung mit den kombinierten Quer- und Längsschnittstichproben und der großen thematischen Breite des DEAS steht damit ein Datensatz zur Verfügung, der eine fundierte Sozialberichterstattung über einen langen Zeitraum hinweg und zu einer Vielzahl alter(n)srelevanter Themen und Fragen ermöglicht. Zugleich erlaubt er wissenschaftlich hochwertige Forschung.
Die erste Erhebung im Jahr 1996 wurde auf Initiative des Bundesministeriums für Familie, Senioren, Frauen und Jugend (BMFSFJ) unter der Federführung der Freien Universität Berlin (Forschungsgruppe Altern und Lebenslauf, Leitung Prof. Martin Kohli) und der Universität Nijmegen (Forschungsgruppe Psychogerontologie, Leitung Prof. Freya Dittmann-Kohli) realisiert. Seit der zweiten Erhebung im Jahr 2002 ist das Deutsche Zentrum für Altersfragen (DZA) mit der Weiterentwicklung, Durchführung und Auswertung des DEAS beauftragt. Die Stichprobenziehung und -pflege sowie die Feldarbeit erfolgen von Studienbeginn an durch das infas Institut für angewandte Sozialwissenschaft 
$\mathrm{GmbH}$, Bonn. Die Daten sowie die dazugehörigen Datendokumentationen (wie Fragebögen und Codebücher) werden durch das Forschungsdatenzentrum des DZA als scientific use files zu Verfügung gestellt. ${ }^{1}$

Auch die jüngste Befragung 2014 folgt dem bisherigen Grundsatz der Wahrung größtmöglicher Konsistenz über die Erhebungsjahre

1 Die Daten des DEAS können für wissenschaftliche Zwecke kostenlos beim Forschungsdatenzentrum des DZA (www.fdz-dza.de) bezogen werden. hinweg - sowohl in den Inhalten als auch im Zugang zu den Befragten. Nur auf diese Weise kann eine Vergleichbarkeit über die Zeit sichergestellt werden. Dennoch bedarf es im Rahmen dieser Vorgabe einer gewissen Flexibilität, um aktuell diskutierte Themen aus Öffentlichkeit, Politik sowie Wissenschaft aufgreifen und vertiefen zu können und - sofern nötig - neueste methodologische Erkenntnisse und Praktiken zur Verbesserung der Datenerhebung und -qualität einfließen zu lassen.

\subsection{Das Stichprobendesign des DEAS}

Der DEAS folgt einem kohortensequenziellen Design (Abbildung 2-1). Durch die Kombination von quer- und längsschnittlichen Stichproben ergeben sich drei Auswertungsmöglichkeiten: Neben der Betrachtung von (1) sozialem Wandel (Vergleich unabhängiger Querschnittsdaten) und (2) individueller Entwicklungsverläufe (Paneldaten) können auch (3) individuelle Entwicklungen im Wandel der Zeit (Kohortenvergleich von Verläufen) untersucht werden. Im vorliegenden Buch werden Fragen des sozialen Wandels behandelt.

Seit 1996 werden im Abstand von sechs Jahren repräsentative Querschnitterhebungen an neuen Stichproben durchgeführt. Dies ermöglicht einen Zeitvergleich auf der Grundlage voneinander unabhängiger Querschnittstichproben. Zielgruppe der Querschnitterhebungen ist jeweils die Wohnbevölkerung in Privathaushalten im Alter von 40 bis 85 Jahren. ${ }^{2}$ Während im
Jahr 1996 nur deutsche Staatsbürgerinnen und Staatsbürger einbezogen wurden, wurde 2002 zudem eine separate Stichprobe der in Deutschland lebenden ausländischen Bevölkerung realisiert. Seit 2008 sind beide Gruppen in einer Bevölkerungsstichprobe zusammengeführt, das heißt unabhängig von der Staatsangehörigkeit stellt seither die in Privathaushalten lebende Wohnbevölkerung Deutschlands die Grundgesamtheit dar. Die Befragung erfolgt ausschließlich in deutscher Sprache. Ergänzend zu diesen Querschnitterhebungen werden seit 2002 jeweils alle Personen, die hierfür schriftlich ihr Einverständnis gegeben haben, für Wiederholungsbefragungen kontaktiert. Wie die Abbildung 2-1 zeigt, wurde nach 2008 die Taktung der Panelerhebungen von sechs auf drei Jahre verkürzt, unter anderem um auf diese Weise Dynamiken individueller Lebensverläufe in der zweiten Lebenshälfte besser abbilden zu können.

2 In den Querschnitterhebungen werden Personen in Gemeinschaftsunterkünften ausgeschlossen, was bezogen auf die im DEAS untersuchte Altersgruppe hauptsächlich ältere Menschen in Pflege- oder Altersheimen betrifft. Laut Mikrozensus 2014 lebt in der Altersgruppe der 40- bis 85-Jährigen nur ein Prozent der Bevölkerung in Gemeinschaftsunterkünften; zu einem substanziellen Anstieg kommt es erst ab dem 80. Lebensjahr (Statistisches Bundesamt 2015a). Somit erlauben die DEAS Querschnittstichproben trotz

Beschränkung auf Privathaushalte für 99 Prozent der Personen in der zweiten Lebenshälfte valide Aussagen. 
Abbildung 2-1: DEAS Stichprobendesign 1996-2014

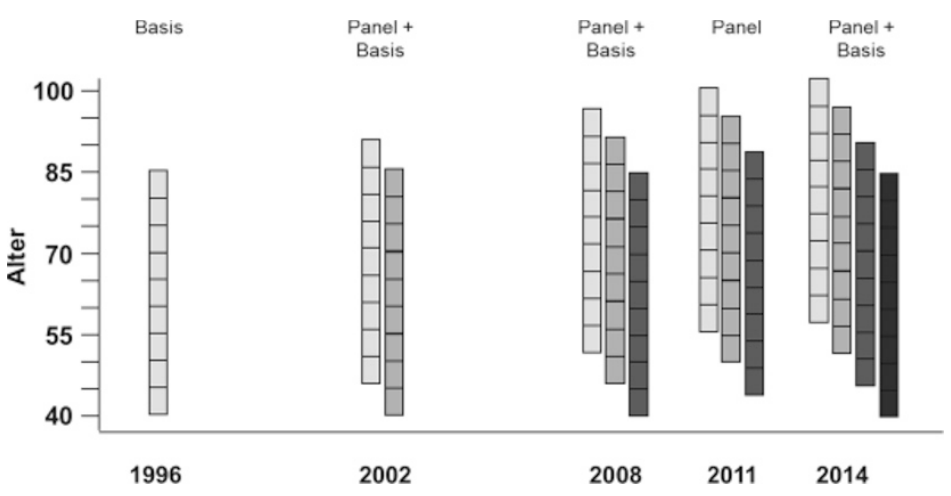

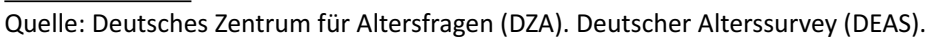

Nach Abschluss der fünften Erhebung im Jahr 2014 verzeichnet der DEAS 20.715 Studienteilnehmerinnen und -teilnehmer, mit denen zwischen 1996 und 2014 insgesamt 33.410 mündliche Interviews geführt wurden. ${ }^{3}$ Zwar hat ein Großteil bislang nur an der jeweiligen Erstbefragung teilgenommen, $(\mathrm{n}=14.091$, darunter 6.002 Teilnehmende an der Erstbefragung im Jahr 2014), doch viele konnten inzwischen auch für wiederholte Befragungen gewonnen werden (zweimal: 2.265; dreimal: 3.041; viermal: 922; fünfmal: 395) - und die Bereitschaft zur mehrfachen Interviewteilnahme steigt. Als Dankeschön

3 Die Angaben beziehen sich auf die gültigen Fälle nach Abschluss der Datenbereinigung im Frühjahr 2015. für ihre Teilnahme haben die Befragten in den ersten Erhebungen Telefonkarten (1996, 2002) und Briefmarkenhefte (2008) erhalten. Seit 2011 werden Aufwandsentschädigungen (Incentives) im Wert von zehn Euro in bar eingesetzt, um die Teilnahmemotivation zu erhöhen (Pforr, Blohm, Blom, Erdel, Felderer, Fräßdorf, Hajek, Helmschrott, Kleinert, Koch, Krieger, Kroh, Martin, Saßenroth, Schmiedeberg, Trüdinger, \& Rammstedt 2015). Gleichzeitig sollen hierüber Ausfälle in den Wiederholungsbefragungen reduziert werden (Laurie \& Lynn 2009) und solche Zielpersonen motiviert werden, die in Umfragen typischerweise unterrepräsentiert sind wie beispielsweise Niedriggebildete oder Alleinstehende (Ryu, Couper, \& Marans 2006).

\subsection{Inhalte}

\subsubsection{Themenschwerpunkte}

Eine Besonderheit des DEAS besteht darin, dass er über einen großen Zeitraum eine sehr große Breite an Themen und Inhalten zu zentralen Lebensbereichen erhebt - und zwar anhand von weitgehend vergleichbaren Instrumenten. Folgende Themenschwerpunkte werden seit 1996 abgedeckt:
- Soziodemografische Grunddaten

- Arbeit und Ruhestand

- Wirtschaftliche Lage, Altersvorsorge und finanzielle Unterstützung

- Soziale Beziehungen (Partnerschaft, Familien- und Generationenbeziehungen, soziale Netzwerke)

- Freizeitaktivitäten, ehrenamtliches Engagement, soziale Unterstützung

- Wohnen und Wohnumfeld 
- Gesundheit, Gesundheitsverhalten, Hilfeund Pflegebedarf

- Einstellungen und Altersbilder

- Lebenszufriedenheit und psychisches Wohlbefinden

Im DEAS wird der Erfassung von biografischen und objektiven Fakten eine ganze Reihe subjektiver Bewertungen und Einstellungen zur Seite gestellt. Dieser Reichtum an Informationen ermöglicht nicht nur eine umfassende Darstellung der Lebenssituation von Menschen in der zweiten Lebenshälfte, sondern kann diese durch deren individuelle Einschätzung ergänzen. Zudem können Wechselwirkungen zwischen Lebensbereichen untersucht werden. Trotz weitgehender inhaltlicher Stabilität über die Befragungsjahre hinweg, finden sich auch Themenbereiche und Fragen, die nur einmalig erhoben wurden oder im Verlauf der Studie hinzugekommen sind. Damit sollen aktuelle Diskurse und Inhalte aufgegriffen werden. So wurden 2014 die Themen soziale Exklusion (vgl. Kapitel 18), nachbarschaftliche Kontakte und lokale Serviceangebote für Ältere (vgl. Kapitel 20 und 21) durch die Aufnahme neuer Erhebungsinstrumente ausgebaut. Der Informationsreichtum des DEAS wird regelmäßig für die Erstellung öffentlichkeitswirksamer Beiträge genutzt, die sich beispielsweise in den Altenberichten der Bundesregierung finden.

Für wiederholt befragte Studienteilnehmerinnen und -teilnehmer ergibt sich ab der Zweitbefragung insofern ein reduziertes Fragenprogramm, als darauf verzichtet wird, stabile Merkmale - wie zum Beispiel das erreichte Bildungsniveau - erneut abzufragen. Zudem werden bereits bekannte Informationen aus vorangegangenen Befragungen als Referenz genutzt, um daran anknüpfend biografische Ereignisse fortzuschreiben (beispielsweise Partnerschaftsbiografie). Dieser Zuschnitt der Befragung auf die individuelle Situation wird durch den umfassenden Einsatz von bekannten Befragteninformationen aus den vorherigen Erhebungen (sogenannte ,Preload-Daten') für die Panelbefragungen möglich.

\subsubsection{Erhebungsmethoden und Befragungsmodule}

Die Datenerhebung im DEAS erfolgt über unterschiedliche Erhebungsverfahren und Befragungsmodule. Mit allen Befragten wird vor Ort ein persönliches mündliches Interview anhand eines standardisierten Fragebogens durchgeführt - in den frühen Erhebungen (1996 und 2002) als paper-pencil-Interview (PAPI) und seit 2008 als Computer unterstütztes Interview (CAPI). Die durchschnittliche Befragungszeit für die Erstbefragungen hat sich über die Befragungsjahre hinweg erhöht und liegt 2014 bei 100 Minuten für Erstbefragte (Median $=96 \mathrm{Mi}$ nuten) und 86 Minuten für wiederholt Befragte (Median $=82$ Minuten). Inbegriffen sind hier Pausen im Verlauf des Interviews, die im Durchschnitt zwischen einer und zwei Minuten betragen. Die durchschnittliche Interviewdauer variiert systematisch mit dem Alter: So liegt sie bei den jüngsten Erstbefragten bei 94 Minuten (40 bis 54 Jahre), in der mittleren Altersgruppe bei 100 Minuten ( 55 bis 69 Jahre) und bei den Ältesten bei 107 Minuten (70 bis 85 Jahre). Trotz der langen Befragungsdauer finden sich auch unter den Älteren keine Hinweise für einen Qualitätsverlust der Daten. So schätzen beispielsweise die Interviewerinnen und Interviewer die Zuverlässigkeit der von den Befragten gemachten Angaben als hoch ein - unabhängig vom Alter der Befragten und der Befragungsdauer. Auch Befragungsabbrüche finden vernachlässigbar selten statt (Schiel, Kerr, Dickmann, \& Aust 2015).

Im Verlauf des mündlichen Interviews werden verschiedene Tests durchgeführt: Seit 2002 wird ein Zahlen-Zeichen-Test zur Messung der psychomotorischen Geschwindigkeit als Teilaspekt der kognitiven Leistungsfähigkeit eingesetzt (Hoyer, Stawski, Wasylyshyn, \& Verhaeghen 2004; Tewes 1994) und ab 2008 ein Lungenfunktionstest als Indikator für die körperliche Gesundheit (Vaz Fragoso, Gahbauer, van Ness, Concato, \& Gill 2008). Etwa 90 Prozent der Befragten nehmen am Lungenfunktionstest teil; die Teilnahmequote beim ZahlenZeichen-Test bewegt sich zwischen 76 und 91 Prozent (vgl. Tabellen 2-1 und 2-3). 
Nach dem mündlichen Interview werden die Befragten gebeten, einen schriftlichen Fragebogen auszufüllen (Drop-Off). Hier werden vor allem Einstellungen und subjektive Einschätzungen erhoben, sensible Fragen wie zum Einkommen und der Gesundheit gestellt und Merkmale erfragt, die keine differenzierte Filterführung benötigen. Auch verschiedene psychologische Skalen werden im Drop-Off erhoben. Während die Teilnahmequote in den ersten beiden Basiserhebungen über 80 Prozent lag, sank sie ab 2008 auf 72 Prozent (vgl. Tabelle 2-1). Bei den Panelbefragten liegt die Ausfüllquote mit jeweils mindestens 80 Prozent deutlich darüber (vgl. Tabelle 2-3), vermutlich aufgrund deren generell größerer Studienbindung und Motivation. Analysen belegen zudem, dass vor allem Frauen, ältere Befragte und Befragte mit einem höheren sozio-ökonomischen Status (Bildung und Einkommen) mit höherer Wahrscheinlichkeit an der schriftlichen Befragung teilnehmen (Schiel et al. 2015). Allerdings ist die Stärke dieser Selektionseffekte recht gering.

Bei Zielpersonen, die aus unterschiedlichen Gründen nicht teilnehmen, wird seit 2008 nach Möglichkeit ein Kurzfragebogen eingesetzt, den entweder die Zielperson selbst beantwortet oder stellvertretend eine Kontaktperson. Ziel ist die Erfassung einiger Basisinformationen wie Wohnform, Gesundheitszustand, Staatsangehörigkeit, Bildung oder Lebenszufriedenheit, um auf diese Weise die (temporären) Ausfälle besser beschreiben zu können. Im Jahr 2014 konnte für 2.198 Zielpersonen der Erstbefragung ein Kurzinterview durchgeführt werden sowie für 308 Personen der Panelstichprobe (Schiel et al. 2015). Das entspricht 14 Prozent beziehungswei- se zwölf Prozent der nicht am Hauptinterview teilnehmenden Zielpersonen der bereinigten Bruttoeinsatzstichproben in der Erst- beziehungsweise Wiederholungsbefragung. In den meisten Fällen wurde das Kurzinterview direkt mit der Zielperson geführt. Die geringe Bereitschaft zur Teilnahme an einem Kurzinterview ist auch aus anderen Altersstudien bekannt (beispielsweise aus dem Survey of Health, Ageing and Retirement in Europe: Börsch-Supan, Brandt, Hunkler, Kneip, Korbmacher, Malter, Schaan, Stuck, \& Zuber 2013).

Ergänzend $\mathrm{zu}$ den Befragungsdaten bietet der DEAS weitere Daten, die das Wohnumfeld der Befragungspersonen auf unterschiedlichen räumlichen Ebenen beschreiben. Für alle Erhebungsjahre liegen Einschätzungen der Interviewenden zur Wohnung und zum Wohnumfeld der Befragten vor. Für die Befragten der Jahre 2002, 2008 und 2014 stehen quartiersbezogene Daten zur sozialstrukturellen Zusammensetzung der Wohngegend, zu Sozialmilieus, Kaufkraft oder zur Arbeitslosigkeit zur Verfügung. Auf Kreisebene können für alle Befragten und alle Erhebungsjahre ausgewählte Indikatoren und Karten zur Raum- und Stadtentwicklung (INKAR-Daten) vom Bundesinstitut für Bau-, Stadt- und Raumforschung genutzt werden wie beispielsweise zu Arbeitsmarkt, Bevölkerung, medizinischer Versorgung, Verkehr oder Wirtschaft.

4 Die Strukturdaten zum Wohnumfeld der Befragten (im Jahr 2002 nur für panelbereite Befragte) wurden von der Firma microm Micromarketing-Systeme und Consult GmbH, Neuss, zur Verfügung gestellt. Für die Befragung des Jahres 2014 liegen auch Wohnumfeldmerkmale der Firma infas $360^{\circ} \mathrm{GmbH}$, Bonn, vor.

\subsection{Die Querschnittstichproben}

\subsubsection{Stichprobenziehung}

Die Querschnittstichproben des DEAS (Basisstichproben) sind Personenstichproben aus Einwohnermelderegistern. Dabei ist die Stich- probenziehung zweistufig angelegt (Schiel et al. 2015; Smid, Hess, \& Gilberg 1997): In der ersten Befragung 1996 wurden entsprechend des ,probability proportional to size'-Ansatzes $290 \mathrm{Ge}$ meinden gezogen. Innerhalb dieser Gebietsein- 
heiten erfolgt seither eine zufällige Ziehung von Zielpersonen aus den Einwohnermelderegistern. Bereits $1996 \mathrm{kam}$ es auf dieser ersten Stufe zu Ausfällen, da fünf Prozent der ausgewählten Gemeinden die Ziehung einer Personenstichprobe verweigert haben (Smid et al. 1997). Im Jahr 2014 fielen aus diesem Grund zwölf Gemeinden aus - allerdings regional zufällig verteilt, weshalb von keinem Qualitätsverlust der Stichprobe auszugehen ist (Schiel et al. 2015).
Seit 2008 werden im Zuge der Aufbereitung der Einwohnermeldeamtsstichproben durch infas diejenigen Personen ausgeschlossen, die bereits in einer der vorangegangenen Erhebungen an der Studie teilgenommen haben. Solche Überschneidungen finden vor allem in kleineren Gemeinden statt: 2008 waren es 278 Personen und 2014 wurden 473 Personen ausgeschlossen (Schiel et al. 2015).

Tabelle 2-1: Merkmale der DEAS-Querschnittstichproben 1996, 2002, 2008, 2014

\begin{tabular}{|c|c|c|c|c|c|}
\hline \multirow[t]{2}{*}{ Befragungsjahr } & \multirow{2}{*}{$\begin{array}{c}1996 \\
\text { Deutsche }\end{array}$} & \multicolumn{2}{|c|}{2002} & 2008 & 2014 \\
\hline & & Deutsche & Ausländer & \multicolumn{2}{|c|}{$\begin{array}{c}\text { Wohnbevölkerung } \\
\text { Deutschland }\end{array}$} \\
\hline \multicolumn{6}{|l|}{ Absolute Zahlen } \\
\hline Unbereinigte Bruttoeinsatzstichprobe ${ }^{1)}$ & 10.608 & 8.826 & 3.225 & 18.822 & 23.984 \\
\hline Bereinigte Bruttoeinsatzstichprobe ${ }^{2)}$ & 9.613 & 8.164 & 2.343 & 17.366 & 22.139 \\
\hline Gültige mündliche Interviews & 4.838 & 3.084 & 586 & 6.205 & 6.002 \\
\hline Gültige schriftliche Fragebögen & 4.034 & 2.787 & 484 & 4.442 & 4.295 \\
\hline Teilnahmen am Zahlen-Zeichen-Test & - & 2.474 & 446 & 5.249 & 4.961 \\
\hline Durchgeführte Lungenfunktionstests & - & - & - & 5.449 & 5.449 \\
\hline Erklärte Panelbereitschaft & 2.873 & 1.964 & 286 & 4.417 & 4.575 \\
\hline \multicolumn{6}{|l|}{ Quoten } \\
\hline Teilnahmequote ${ }^{3)}$ & 50,3 & 37,8 & 25,0 & 35,7 & 27,1 \\
\hline Schriftlicher Fragebogen ${ }^{4)}$ & 83,4 & 90,4 & 82,6 & 71,6 & 71,6 \\
\hline Zahlen-Zeichen-Test ${ }^{4)}$ & - & 80,2 & 76,1 & 84,6 & 82,7 \\
\hline Lungenfunktionstest ${ }^{4)}$ & - & - & - & 87,8 & 90,8 \\
\hline Panelbereitschaftsquote ${ }^{4)}$ & 59,4 & 63,7 & 48,8 & 71,2 & 76,2 \\
\hline \multicolumn{6}{|l|}{ Sonstiges } \\
\hline Erhobene Geburtsjahrgänge & $1911-56$ & $1917-62$ & $1917-62$ & $1923-68$ & $1929-74$ \\
\hline Alter bei Befragung (Jahre) & $40-85$ & $40-85$ & $40-85$ & $40-85$ & $40-85$ \\
\hline Durchschnittliche Interviewdauer (Min.) & 67 & 82 & 82 & 83 & 100 \\
\hline Seitenzahl schriftlicher Fragebogen & 28 & 32 & 32 & 36 & 36 \\
\hline Erhebungszeitraum (Monate/Jahr) & $1-7 / 1996$ & $2-11 / 2002$ & $2-11 / 2002$ & $4-11 / 2008$ & $4-11 / 2014$ \\
\hline
\end{tabular}

Quellen: Eigene Zusammenstellung bzw. Berechnung basierend auf den DEAS-scientific use files (Stand: Frühjahr 2015) sowie Engstler \& Motel-Klingebiel (2010); Engstler \& Wurm (2006); Klaus \& Engstler (2012); Künemund (2000); Schiel, Hess \& Gilberg (2003); Schiel \& Smid (2009); Schiel, Dickmann \& Aust (2011); Schiel, Kerr, Dickmann \& Aust (2015). Anmerkungen: 1) Zufallsstichprobe aus dem von den Einwohnermeldeämtern gelieferten Adressdatensatz; 2) Unbereinigte Bruttoeinsatzstichprobe abzüglich neutraler Ausfälle, das heißt Personen, die bereits verstorben sind, im Heim leben, ins Ausland oder unbekannt verzogen sind, nicht ausreichend deutsch sprechen oder aus anderen Gründen nicht zur Zielgruppe gehören; 3) Anteil gültiger mündlicher Interviews an bereinigter Bruttoeinsatzstichprobe; 4) Anteil an gültigen mündlichen Interviews. 
Die Querschnittstichproben sind seit der ersten Erhebung disproportional nach Alter, Geschlecht und Landesteil geschichtet: Die Ziehung der Bruttostichprobe erfolgt so, dass sich erwarten lässt, dass sich die realisierten Interviews zu etwa gleichen Teilen auf drei Altersgruppen ( 40 bis 54,55 bis 69,70 bis 85 Jahre) sowie auf Männer und Frauen verteilen. Zwei Drittel der Befragten sollen in den alten und ein Drittel in den neuen Bundesländern wohnen. Demzufolge sind Ältere, Männer und Befragte in Ostdeutschland in den geschichteten Zufallsstichproben überproportional vertreten. Diese Schichtung verfolgt das Ziel, auch in Wiederholungsbefragungen noch ausreichend große Fallzahlen für Personengruppen mit relativ geringen Bevölkerungsanteilen sicherzustellen (wie beispielsweise hochaltrige Männer in Ostdeutschland). Aus diesem Grund wird seit 2008 eine Gesamtfallzahl von etwa 6.000 Personen pro Querschnittstichprobe angestrebt, während die realisierten Stichprobenumfänge in den Vorjahren geringer ausfielen (vgl. die Anzahl gültiger Interviews in Tabelle 2-1).

\subsubsection{Teilnahmebereitschaft}

Während die Teilnahmequote - der Anteil realisierter gültiger Interviews an der bereinigten Bruttoeinsatzstichprobe ${ }^{5}$ - zu Beginn der Studie 1996 bei 50 Prozent lag, ist sie auf 27 Prozent bei der Querschnittsstichprobe im Jahr 2014 zurückgegangen. Die sinkende Teilnahmebereitschaft im DEAS ist eingebettet in einen generell rückläufigen Trend zur Teilnahme an sozialwissenschaftlichen Studien in westlichen Industrienationen (Aust \& Schröder 2009; Brick \& Williams 2013; de Leeuw \& de Heer 2002), der in Deutschland besonders stark ausgeprägt ist (Stoop, Billiet, Koch, \& Fitzgerald 2010). Maßnahmen, wie eine umfassende postalische Vorabinformation der Zielpersonen (Anschrei-

5 Zufallsstichprobe aus dem von den Einwohnermeldeämtern gelieferten Adressdatensatz abzüglich neutraler Ausfälle. Um neutrale Ausfälle handelt es sich, wenn Personen bereits verstorben sind, im Heim leben, ins Ausland oder unbekannt verzogen sind, nicht ausreichend deutsch sprechen oder aus anderen Gründen nicht zur Zielgruppe gehören. ben von infas, Begleitschreiben des BMFSFJ und des DZA) sowie die Erhöhung des finanziellen Wertes des Incentives zwischen 2008 und 2014 haben diese negative Entwicklung möglicherweise abschwächen, aber nicht aufhalten können. Folglich war die Ziehung und Bearbeitung immer größerer Einsatzstichproben notwendig (vgl. Tabelle 2-1) - ein Trend, der sich zukünftig fortsetzen könnte.

Entsprechend der in Deutschland generell geringeren Teilnahmequote an freiwilligen Befragungen ist auch die Teilnahmequote am DEAS niedriger als in ähnlich gelagerten Alterssurveys anderer europäischer Länder: So lag die Erstteilnahmequote in der niederländischen LASA-Studie (Altersspanne 55 bis 85 Jahre) im Jahr 2002 bei 55 Prozent (Huisman, Poppelaars, van der Horst, Beekman, Brug, van Tilburg, \& Deeg 2011), in der europäisch-vergleichenden SHARE-Studie (Alter 50+) 2004/2005 über alle Länder hinweg bei 62 Prozent (Börsch-Supan et al. 2013) und in der irischen Studie TILDA (Alter $50+$ ) im Jahr 2010 bei 62 Prozent (Nolan, O’Regan, Dooley, Wallace, Hever, Cronin, Hudson, \& Kenny 2014). Gleichzeitig ähnelt die DEAS-Teilnahmequote anderen deutschlandweiten Studien mit durchschnittlich jüngerer Zielgruppe: Die Erstteilnahmequote unter 25bis 27-Jährigen beziehungsweise 35- bis 37-Jährigen in der pairfam-Studie 2008 lag bei 33 beziehungsweise 32 Prozent (Brüderl, Schmiedeberg, Castiglioni, Arránz Becker, Buhr, Fuß, Ludwig, Schröder, \& Schumann 2015), im ALLBUS 2012 für vergleichbare Altersgruppen bei 38 Prozent (Wasmer, Blohm, Walter, Scholz, \& Jutz 2014) und in der SOEP-Aufstockungsstichprobe 2012 bei den über 16-Jährigen bei 35 Prozent (TNS infratest Sozialforschung 2013).

\subsubsection{Stichprobenselektivität}

Wie gut sich die Ergebnisse basierend auf den Querschnittstichproben für die Gesamtbevölkerung generalisieren lassen, hängt weniger von der allgemeinen Teilnahmebereitschaft ab, sondern vielmehr davon, ob und wie selektiv die Teilnahme für bestimmte Bevölkerungsgruppen ausfällt. Für die drei Schichtungsvariablen (Alter, Geschlecht und Landesteil) sowie die 
Größe des Wohnortes der Zielperson lässt sich prüfen, ob sich Teilnehmerinnen und -teilnehmer signifikant von denjenigen unterscheiden, die nicht teilgenommen haben. Für die drei Querschnittserhebungen 2002, 2008 und 2014 zeigen sich hierbei geringfügige Selektivitäten (Schiel et al. 2015; Schiel et al. 2003; Schiel \& Smid 2009): (1) Frauen haben eine etwas geringere Teilnahmewahrscheinlichkeit als Männer ein Effekt entgegengesetzt zu Umfragen innerhalb der jüngeren Bevölkerung, in der Frauen tendenziell besser erreichbar sind als Männer (Schnell 1997). Möglicherweise sind sie im höheren und hohen Alter etwas misstrauischer, insbesondere wenn sie alleine leben. (2) Mit zunehmender Einwohnerzahl des Wohnortes der Zielperson sinkt die Teilnahmebereitschaft. Vor allem die geringere Teilnahmequote in Großstädten ist ein aus anderen Umfragen bekannter Befund, der unter anderem mit höherer Mobilität und damit einhergehender schlechterer Erreichbarkeit begründet wird. (3) Im DEAS 2008 fällt die Teilnahmewahrscheinlichkeit in den neuen Bundesländern etwas geringer aus als in den alten Bundesländern, in den anderen Erhebungsjahren zeigen sich keine Unterschiede. (4) Im Vergleich der Altersgruppen ist die Teilnahmewahrscheinlichkeit unter den 55- bis 69-Jährigen am höchsten. Es ist davon auszugehen, dass Personen im (jüngeren) mittleren Erwachsenenalter (Altersgruppe der $40-$ bis 54-Jährigen) aufgrund oft gleichzeitiger familiärer und beruflicher Verpflichtungen zeitlich sehr eingeschränkt sind und zudem recht mobil, was ihre Verfügbarkeit reduziert. Hingegen ist die etwas geringere Teilnahmequote unter den ältesten Zielpersonen (70- bis 85-Jährige) vor allem durch eine gesundheitsbedingt reduzierte Befragbarkeit zu erklären (Motel-Klingebiel, Klaus, \& Simonson 2014). Insgesamt erweisen sich jedoch die Selektivitäten hinsichtlich der diskutierten demografischen Merkmale als recht gering: Die durch sie aufgeklärte Varianz in der Teilnahmebereitschaft bewegt sich lediglich zwischen zwei und vier Prozent (Schiel et al. 2015; Schiel et al. 2003; Schiel \& Smid 2009).

\subsubsection{Datengewichtung}

Zum Ausgleich der disproportionalen Stichprobenziehung wurde für jede Basisstichprobe eine Datengewichtung vorgenommen. Diese erfolgt als Randanpassung der Stichprobe an die relative Häufigkeit der zwölf Merkmalskombinationen der Stichprobenschichtung aus Altersgruppe, Geschlecht und Landesteil in der amtlichen Bevölkerungsstatistik. Referenz hierfür ist jeweils der Mikrozensus. ${ }^{6}$ Die Gewichtung wurde getrennt für die mündlichen Interviews und die schriftliche Befragung vorgenommen. Sie gleicht zudem Selektivitäten in der Befragungsteilnahme nach diesen drei Merkmalen aus. Im vorliegenden Buch kommen diese Querschnittsgewichte für deskriptive Darstellungen zum Einsatz.

\subsubsection{Repräsentativität}

Für Hinweise auf weitere Selektionsmechanismen jenseits der betrachteten Schichtungsvariablen sowie zur Bewertung der Stichprobenqualität unter Verwendung der Querschnittgewichtung werden beispielhaft für 2014 die ungewichteten und gewichteten Anteile weiterer Merkmale des DEAS den entsprechenden Anteilen aus der amtlichen Statistik gegenübergestellt. Auf diese Weise wird geprüft, ob nach der Gewichtung bestimmte Bevölkerungsgruppen im DEAS systematisch unteroder überrepräsentiert sind. In Tabelle 2-2 sind diese Zahlen zusammengestellt.

Im Allgemeinen zeigt sich, dass die Verteilungen im DEAS unter Verwendung der Gewichtung nahe an den Verteilungen in der amtlichen Statistik liegen (vgl. Tabelle 2-2). Lediglich leichte Abweichungen bleiben bestehen. So zeigt

6 Für frühere Versionen der Querschnittstichproben der DEAS-Erhebungen 1996 bis 2008 wurde die Bevölkerungsfortschreibungsstatistik als Gewichtungsreferenz verwendet. Diese wird nun für alle Querschnittstichproben durch den Mikrozensus ersetzt, der zielgenauer die für den DEAS relevante Grundgesamtheit der am Ort der Hauptwohnung lebenden Bevölkerung in Privathaushalten repräsentiert. Wir danken dem Statistischen Bundesamt für die Bereitstellung der Mikrozensus-Referenztabellen. 
sich - wie auch aus vorangegangenen Befragungen bekannt (Engstler \& Motel-Klingebiel, 2010) - eine Tendenz zur Überrepräsentation von Familienhaushalten und Befragten aus der Mittelschicht: Die gewichteten Anteile von Verheirateten und Personen in Haushalten mit vier oder mehr Mitgliedern liegen im DEAS geringfügig über den Werten aus dem Mikrozensus. Ebenso fallen die Anteile von Personen mit höherer Schulbildung, Angestellten und Beamten etwas höher aus. Darüber hinaus besteht ein für die Befragung von älteren und alten Bevölkerungsgruppen kaum zu vermeidender Gesundheitsbias, der aber erst im höheren Alter auftritt. So zeigt sich bei den unter 65-Jährigen noch keine Untererfassung von Schwerbehinderten im DEAS (Tabelle 2-2) - und auch der geringere Anteil Pflegebedürftiger mit Pflegestufe geht weitgehend auf Differenzen bei den über 80-Jährigen zwischen DEAS und Pflegestatistik zurück (ohne Abbildung).

Personen mit Migrationshintergrund stellen inzwischen einen wesentlichen Teil der Bevölkerung in der zweiten Lebenshälfte dar. Ihr Anteil unter den 40 - bis 85 -Jährigen beträgt laut $\mathrm{Mi}$ krozensus knapp 15 Prozent (Tabelle 2-2). Der gewichtete DEAS liegt mit zehn Prozent etwas darunter. Diese Unterschätzung gilt für Männer gleichermaßen wie für Frauen und sie fällt in den beiden jüngsten Altersgruppen größer aus als in der ältesten DEAS-Befragtengruppe der 70- bis 85-Jährigen (nicht in Tabelle 2-2 gezeigt). Für das bekannte Phänomen der geringeren Teilnahmequote von Personen mit Migrationshintergrund werden in der Literatur verschiedene Gründe genannt, die von Sprachschwierigkeiten bis zum kulturellen Hintergrund und spezifischen Migrationserfahrungen reichen (Deding, Fridberg, \& Jakobsen 2008; Stadler 2009; Blohm \& Diehl 2001; Baykara-Krumme 2013). So setzen beispielsweise konservative Werthaltungen, kulturelle Distanz zum Interviewenden oder auch unsicherer rechtlicher oder wirtschaftlicher Status die Kooperationsbereitschaft herab. Entsprechend ist davon auszugehen, dass vor allem (rechtlich, sprachlich, wirtschaftlich und sozial) schlechter integrierte Migrantinnen und Migranten im DEAS unterdurchschnittlich vertreten sind.

Trotz der genannten Abweichungen weist die Verteilung zentraler sozio-demografischer Merkmale im DEAS eine große Ähnlichkeit mit der Verteilung der amtlichen Statistik auf. Entsprechend stellen die gewichteten Querschnittsstichproben eine sehr gute Datengrundlage zur Untersuchung der Lebenssituation der Menschen in der zweiten Lebenshälfte dar. 
Tabelle 2-2: Verteilung ausgewählter Merkmale in der DEAS-Basisstichprobe 2014 und der amtlichen Statistik

\begin{tabular}{|c|c|c|c|c|}
\hline \multirow{2}{*}{ Merkmal } & \multicolumn{2}{|c|}{ Basisstichprobe 2014} & \multirow{2}{*}{$\begin{array}{l}\text { Amtliche } \\
\text { Statistik }\end{array}$} & \multirow{2}{*}{$\begin{array}{l}\text { Quelle der amtlichen } \\
\text { Statistik }\end{array}$} \\
\hline & Ungewichtet & Gewichtet & & \\
\hline \multicolumn{5}{|l|}{ Familienstand } \\
\hline ledig & 8,9 & 10,1 & 12,5 & \multirow{4}{*}{$\begin{array}{c}\text { Statistisches } \\
\text { Bundesamt (2015), } \\
\text { Mikrozensus } 2014\end{array}$} \\
\hline verheiratet $^{1}$ & 70,1 & 69,8 & 66,6 & \\
\hline geschieden ${ }^{2}$ & 10,9 & 11,2 & 11,0 & \\
\hline verwitwet ${ }^{3}$ & 10,1 & 8,9 & 9,9 & \\
\hline \multicolumn{5}{|l|}{ Haushaltsgröße } \\
\hline 1 & 19,9 & 19,4 & 23,0 & \multirow{4}{*}{$\begin{array}{c}\text { Statistisches } \\
\text { Bundesamt (2015), } \\
\text { Mikrozensus } 2014\end{array}$} \\
\hline 2 & 54,5 & 48,6 & 46,6 & \\
\hline 3 & 13,4 & 15,4 & 15,0 & \\
\hline $4+$ & 12,2 & 16,6 & 15,5 & \\
\hline \multicolumn{5}{|l|}{ Höchster Schulabschluss } \\
\hline ohne oder unbekannt & 2,0 & 2,1 & 4,1 & \multirow{5}{*}{$\begin{array}{c}\text { Statistisches } \\
\text { Bundesamt (2015), } \\
\text { Mikrozensus 2014 }\end{array}$} \\
\hline Volks-/Hauptschule & 30,8 & 31,9 & 40,6 & \\
\hline Polytechnische Oberschule & 19,6 & 12,4 & 10,7 & \\
\hline Realschule, Mittlere Reife & $20,4^{4}$ & $24,6^{4}$ & 20,1 & \\
\hline Abitur, Fachhochschulreife & 27,2 & 29,0 & 24,6 & \\
\hline \multicolumn{5}{|c|}{ Berufliche Stellung der 40- bis 64-jährigen Erwerbstätigen } \\
\hline Arbeiter & 18,6 & 17,7 & 22,2 & \multirow{4}{*}{$\begin{array}{c}\text { Statistisches } \\
\text { Bundesamt (2015), } \\
\text { Mikrozensus 2014 }\end{array}$} \\
\hline Angestellte & 61,7 & 62,4 & 59,2 & \\
\hline Beamte & 8,3 & 8,8 & 5,5 & \\
\hline Selbstständige ${ }^{5}$ & 11,4 & 11,1 & 13,0 & \\
\hline \multicolumn{5}{|l|}{ Erwerbsbeteiligung } \\
\hline erwerbstätig & 46,5 & 53,8 & 54,0 & \multirow{2}{*}{$\begin{array}{c}\text { Statistisches } \\
\text { Bundesamt (2015), } \\
\text { Mikrozensus 2014 }\end{array}$} \\
\hline nicht erwerbstätig & 53,5 & 46,2 & 46,0 & \\
\hline \multicolumn{5}{|c|}{ Äquivalenzeinkommen (Median, Euro/Monat) } \\
\hline 50- bis 64-Jährige & 1.750 & 1.867 & $1.718^{6}$ & \multirow{2}{*}{$\begin{array}{c}\text { Statistisches } \\
\text { Bundesamt (2015a), } \\
\text { EU-SILC } 2013\end{array}$} \\
\hline 65-Jährige und Ältere & 1.417 & 1.500 & $1.492^{6}$ & \\
\hline \multicolumn{5}{|l|}{ Anteil Schwerbehinderter } \\
\hline 45- bis 54-Jährige & 8,2 & 8,1 & 6,8 & \multirow{3}{*}{$\begin{array}{c}\text { Statistisches } \\
\text { Bundesamt (2014), } \\
\text { Statistik schwer- } \\
\text { behinderter Menschen }\end{array}$} \\
\hline 55- bis 64-Jährige & 15,8 & 16,0 & 15,2 & \\
\hline 65-Jährige und Ältere & 19,3 & 19,4 & 24,3 & \\
\hline
\end{tabular}




\begin{tabular}{|c|c|c|c|c|}
\hline \multirow{2}{*}{ Merkmal } & \multicolumn{2}{|c|}{ Basisstichprobe 2014} & \multirow{2}{*}{$\begin{array}{l}\text { Amtliche } \\
\text { Statistik }\end{array}$} & \multirow{2}{*}{$\begin{array}{c}\text { Quelle der amtlichen } \\
\text { Statistik }\end{array}$} \\
\hline & Ungewichtet & Gewichtet & & \\
\hline \multicolumn{5}{|c|}{ Pflegebedürftigkeit der 60- bis unter 85-Jährigen } \\
\hline ohne & 97,9 & 97,9 & 93,8 & \multirow{2}{*}{$\begin{array}{c}\text { Statistisches } \\
\text { Bundesamt (2015b), } \\
\text { Pflegestatistik 2013 }\end{array}$} \\
\hline mit & 2,1 & 2,1 & 6,2 & \\
\hline \multicolumn{5}{|c|}{ Staatsangehörigkeit } \\
\hline deutsch & 96,6 & 95,8 & 92,3 & \multirow{2}{*}{$\begin{array}{c}\text { Statistisches } \\
\text { Bundesamt (2015), } \\
\text { Mikrozensus 2014 }\end{array}$} \\
\hline nicht-deutsch & 3,4 & 4,2 & 7,7 & \\
\hline \multicolumn{5}{|c|}{ Migrationshintergrund } \\
\hline ohne & 91,9 & 90,2 & 85,0 & \multirow{2}{*}{$\begin{array}{c}\text { Statistisches } \\
\text { Bundesamt (2015), } \\
\text { Mikrozensus 2014 }\end{array}$} \\
\hline mit & 8,1 & 9,8 & 15,0 & \\
\hline
\end{tabular}

Quellen: DEAS Basisstichprobe 2014 ( $n=2.244-6.002)$, Statistisches Bundesamt; wenn nicht anders angegeben: 40bis 85 -Jährige.

Anmerkungen: 1) einschließlich eingetragener Lebenspartnerschaft; 2) einschließlich aufgehobener Lebenspartnerschaft; 3) einschließlich verstorbener eingetragener Lebenspartnerinnen und -partner; 4) einschließlich weiterführender Schulabschluss im Ausland; 5) einschließlich mithelfende Familienangehörige; 6) ein Zwölftel des Jahreseinkommens.

\subsection{Die Panelstichproben}

\subsubsection{Realisierung von Wiederholungsbefragungen}

Im Rahmen jeder querschnittlichen Erstbefragung werden die Befragten um ihr schriftliches Einverständnis gebeten, Namen und Adresse speichern zu dürfen. Diese sogenannte Panelbereitschaft - die datenschutzrechtlich die Grundlage für eine erneute Kontaktaufnahme zwecks Durchführung von Wiederholungsbefragungen ist - ist zwischen 1996 und 2014 stetig angestiegen: von 59 Prozent auf 76 Prozent (vgl. Tabelle 2-1).

Wiederholungsbefragungen der Studienteilnehmerinnen und -teilnehmer werden seit der zweiten Erhebung im Jahr 2002 - parallel zu den Erstbefragungen - durchgeführt. Um die mit dem Alter steigende Dynamik biografischer Ereignisse in zentralen Bereichen wie der
Gesundheit besser abbilden zu können, wurde nach 2008 die Taktung zwischen den Wiederholungsbefragungen von sechs Jahren auf drei Jahre verkürzt. Weiterhin wurden - um die Panelbereitschaft und Wiederteilnahmequoten $\mathrm{zu}$ erhöhen - zwischen den Befragungen Panelpflegen durchgeführt: $\mathrm{Zu}$ Beginn der Studie unregelmäßig, ab 2012 in jährlichem Abstand. Darüber wird einerseits der Adressbestand aktualisiert, was die Erreichbarkeit der Befragten verbessert. Andererseits sollen die Befragten durch das regelmäßige Versenden von Grußkarten, Ergebnis- und Informationsbroschüren stärker an die Studie gebunden werden, um auf diese Weise deren Motivation zur weiteren Teilnahme zu erhöhen.

In jeder Wiederholungsbefragung werden als Panelstichprobe alle Befragten angeschrieben, die nach der jeweils letzten Panelpflege als noch 
lebend bekannt sind, nicht ins Ausland verzogen sind und für die weiterhin die Panelbereitschaft vorliegt (unbereinigte Bruttoeinsatzstichprobe, vgl. Tabelle 2-3). Kontaktiert werden auch diejenigen, die einmal oder mehrfach nicht an Wiederholungsbefragungen teilgenommen haben, solange ihre Panelbereitschaft nicht widerrufen wurde. Erfahrungen verschiedener (Alters-)Studien haben gezeigt, dass sich die Zulassung mehrfachen Aussetzens positiv auf die Qualität der Stichprobe auswirkt (Michaud, Kapteyn, Smith, \& van Soest 2011; Weir, Faul, \& Langa 2011): Es wird erstens die Panelmortalität herabgesetzt und zweitens können Selektionseffekte gemindert werden, die durch den überproportionalen Ausfall von Personen verursacht werden, die lediglich vorübergehend nicht erreichbar oder befragbar sind, etwa weil sie sich temporär im Ausland aufhalten oder im Krankenhaus sind.

\subsubsection{Panelmortalität}

Die in Tabelle 2-3 berichteten Teilnahmequoten in den Längsschnittstichproben belegen hohe Ausfälle in der jeweils ersten Wiederholungsbefragung, die sich in den weiteren Befragungsjahren abschwächen - ein Muster, das auch aus anderen Studien bekannt ist (SHARE: Blom \& Schröder 2011). Die Verkürzung der Paneltaktung im DEAS nach 2008 auf drei Jahre hat sich positiv auf die Wiederteilnahme ausgewirkt: So liegt die Wiederbefragungsquote der Basisstichprobe $2008 \mathrm{im} \mathrm{Jahr} 2011$ mit 46,1 Prozent über der Quote der deutschen SHARE-Stichprobe (41 Prozent), die eine vergleichbare Altersgrup- pe (50-Jährige und älter) sogar alle zwei Jahre befragt (Schröder 2008).

Eine Reihe von Analysen belegen eine gewisse Selektivität der Ausfälle (Schiel et al. 2015; Engstler \& Motel-Klingebiel 2010; Schiel \& Smid 2009): Während sich Geschlecht, Wohnort (Verstädterungsgrad und Landesteil) sowie Familien- und Haushaltskonstellation nicht oder nicht durchweg konsistent auf die erneute Teilnahme auswirken, findet sich ein über die Panelerhebungen und Teilnahmekonstellationen hinweg stabiler negativer Alterseffekt: Je älter die Befragten zur Erstbefragung sind, desto unwahrscheinlicher ist ihre erneute Teilnahme. Dahinter steht eine mit dem Alter zunehmende Verschlechterung der Erreichbarkeit oder Befragbarkeit aufgrund gesundheitlicher Einschränkungen, temporärer Krankenhausaufenthalte, Umzüge in Betreuungs- oder Pflegeeinrichtungen oder auch das Versterben der Befragten. Weiterhin beeinflussen hohe Bildung, hohes Einkommen, gute Gesundheit und ein großes Netzwerk den Verbleib in der Längsschnittstichprobe positiv - Selektionseffekte, die sich auch in anderen Studien finden (Banks, Muriel, \& Smith 2011; Schröder 2008). Für den DEAS zeigt sich, dass Alters-, Gesundheits- und Bildungseffekte bei dreijährigem gegenüber sechsjährigem Befragungsabstand an Stärke verlieren (Klaus \& Engstler 2012; Engstler \& MotelKlingebiel 2010). Damit reduziert die verkürzte Taktung zwischen den Befragungen nicht nur die Ausfallwahrscheinlichkeit, sondern auch die sozialstrukturell und gesundheitsbedingte Teilnahmeselektivität, was insgesamt eine Verbesserung der Qualität der Panelstichproben mit sich bringt. 


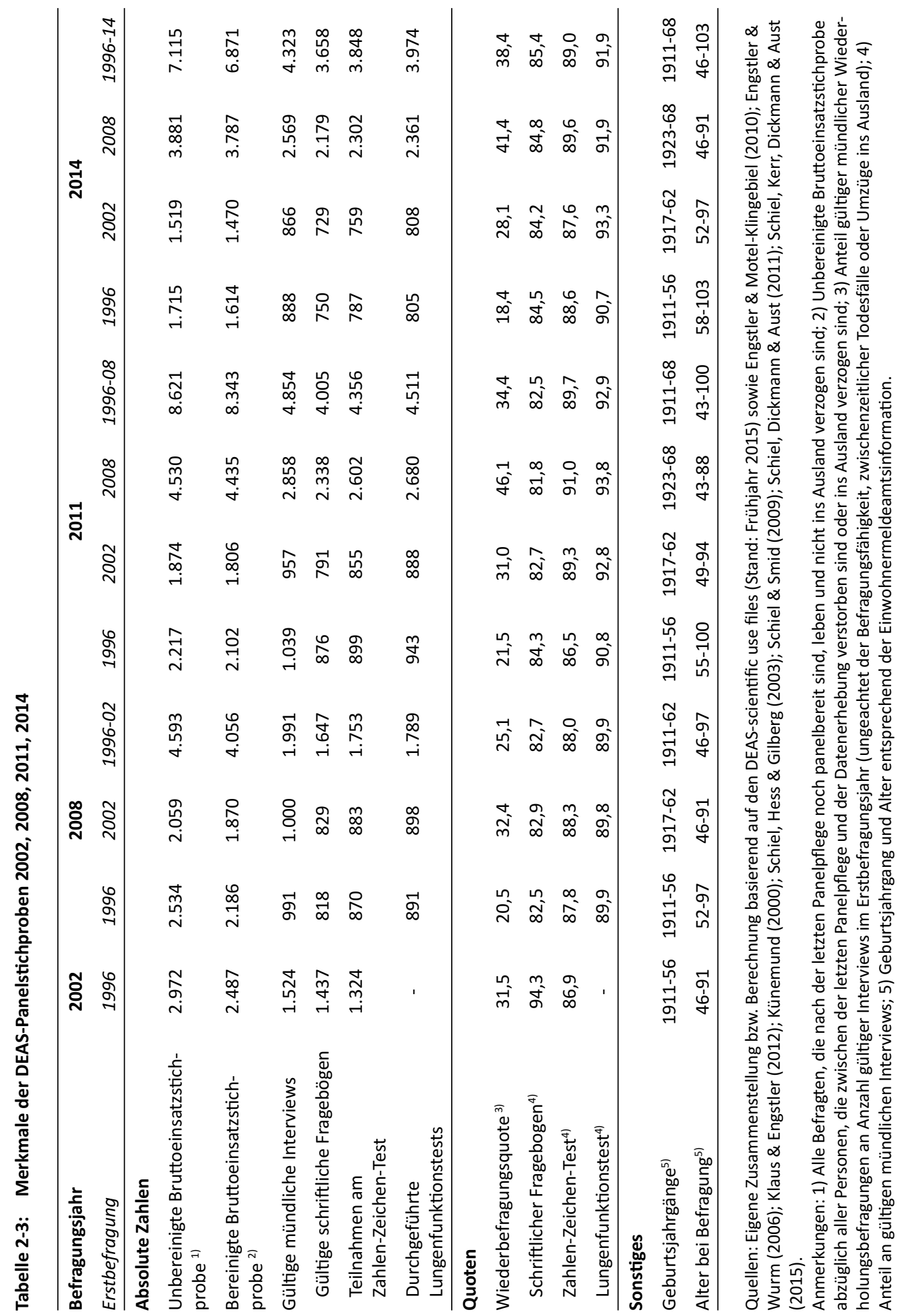




\subsection{Auswertungen im vorliegenden Buch}

Das vorliegende Buch liefert eine umfassende Beschreibung des Wandels der Lebenssituationen von Personen in der zweiten Lebenshälfte zwischen 1996 und 2014. Es wird Fragen des sozialen Wandels nachgegangen. Datengrundlage bilden die Querschnitterhebungen der Jahre 1996, 2002, 2008 und 2014, das heißt die jeweils neu gezogenen Basisstichproben der 40bis 85-Jährigen. Neben Veränderungen über die Zeit wird auch Zusammenhängen zwischen inhaltlich interessierenden Indikatoren und folgenden Gruppierungsvariablen nachgegangen.

\subsubsection{Gruppierungsvariablen}

In allen Kapiteln wird der Zusammenhang der untersuchten Indikatoren mit Geschlecht und Altersgruppen geprüft. Je nach spezifischer Fragestellung werden in einzelnen Kapiteln weitere Gruppenvergleiche in den Mittelpunkt gerückt, vorrangig Vergleiche nach Landesteil und nach Bildungsgruppen.

Geschlecht. Das Geschlecht der Befragungsperson wird - beruhend auf der Einwohnermeldeamtsinformation oder der Interviewerangabe dichotom als männlich oder weiblich ausgewiesen.

Altersgruppen. In manchen Kapiteln werden ergänzend oder alternativ zu den drei Altersgruppen, die jeweils 15 Jahre abdecken und der Schichtung der Basisstichprobe entsprechen (40- bis 54-Jährige, 55 - bis 69-Jährige, 70 - bis 85-Jährige) folgende Sechsjahres-Altersgruppen verwendet: 42 - bis 47 -Jährige, 48 - bis 53 -Jährige, 54- bis 59-Jährige, 60- bis 65-Jährige, 66- bis 71-Jährige, 72- bis 77-Jährige, 78- bis 83-Jährige. Dieses Vorgehen ermöglicht in Verbindung mit den Sechsjahres-Abständen zwischen den Querschnittstichproben einen Kohortenvergleich, da auf diese Weise Gruppen nicht überlappender Geburtsjahrgänge definiert und miteinander verglichen werden können.

Landesteil/Region. Hier wird zwischen den alten Bundesländern (inklusive dem früheren Westteil von Berlin) und den neuen Bundesländern (inklusive dem früheren Ostteil Berlins) unterschieden.
Bildungsgruppen. Basierend auf der international vergleichbaren ISCED-Klassifizierung (UNESCO 2006) werden drei Bildungsgruppen unterschieden: In der Gruppe der Niedriggebildeten sind Befragte ohne abgeschlossene berufliche Ausbildung zu finden (ISCED 0-2). Befragte mit mittlerem Bildungsniveau sind diejenigen mit Abschluss einer betrieblichen oder berufsbildend-schulischen Ausbildung. Eingeschlossen sind hier auch Befragte mit höherem allgemeinbildenden Schulabschluss ohne abgeschlossene Berufsausbildung (ISCED 3-4). Als hochgebildet gelten Befragte mit abgeschlossenem Studium einer Fachhochschule, Universität oder Hochschule oder mit abgeschlossener Aufstiegsfortbildung (Fach-, Meister-, Technikerschule, Berufs- oder Fachakademie (ISCED 5-6)).

\subsubsection{Analysestrategie}

Die statistische Auswertung der Daten erfolgt in allen Kapiteln mittels multivariater Analyseverfahren. Unterschiede zwischen Erhebungsjahren und zwischen Befragtengruppen werden so auf ihre statistische Bedeutsamkeit hin überprüft. Für dichotome und dichotomisierte Indikatoren kommt die binäre logistische Regression zum Einsatz. Multinomiale logistische Regressionen werden für kategoriale Indikatoren verwendet, während Ordered-Logit-Regressionen für ordinal-skalierte Indikatoren genutzt werden. Bei intervall-skalierten Indikatoren werden lineare Regressionen oder Varianzanalysen angewandt. In allen Regressionsmodellen werden die Schichtungsmerkmale der Stichprobe - Altersgruppe, Geschlecht und Landesteil kontrolliert. Weitere Gruppierungsvariablen, wie beispielsweise Bildung oder weitere regionale Differenzierungen, werden aufgenommen, sofern deren Signifikanz geprüft werden soll. Weiterhin werden Interaktionsterme auf Signifikanz getestet, wenn entsprechende Fragestellungen beantwortet werden sollen - zum Beispiel, ob sich Geschlechtsunterschiede in den drei Altersgruppen unterscheiden. Geht es um die Fra- 
ge, ob sich der Wandel über die Erhebungsjahre nach Altersgruppe unterscheidet, wird eine Interaktion aus Erhebungsjahr und den Sechsjahres-Altersgruppen (42- bis 47-Jährige, 48- bis 53-Jährige, 54- bis 59-Jährige, 60- bis 65-Jährige, 66- bis 71-Jährige, 72- bis 77-Jährige, 78- bis 83-Jährige) geprüft. Dadurch wird erreicht, dass sich die Geburtsjahrgänge zu den einzelnen Erhebungszeitpunkten nicht überlappen.

Zur Ergebnisdarstellung im Text und in den Abbildungen werden gewichtete, erhebungsund gruppenspezifische Maße genutzt. Je nach Skalenniveau der Indikatoren werden prozentuale Häufigkeiten oder Mittelwerte berichtet. Zur Gewichtung der deskriptiven Häufigkeitsverteilungen oder Mittelwerte werden in den meisten Kapiteln die Querschnittsgewichte - je nach Indikator für die Daten des Interviews oder des schriftlichen Fragebogens - genutzt (vgl. Abschnitt 2.4.4). In einem Tabellenanhang (www.deutscher-alterssurvey.de sowie auf der Produktseite des Buches auf www.springer.com) werden ergänzend hierzu die auf diese Weise gewichteten Befunde für alle verwendeten Indikatoren zusammengestellt - getrennt für jedes Erhebungsjahr sowie für die zwölf Zellen, die sich aus der Kombination der Schichtungsvariablen ergeben: Landesteil (alte versus neue Bundesländer), Frauen und Männer sowie drei Alters- gruppen (40- bis 54-Jährige, 55- bis 69-Jährige, 70- bis 85-Jährige). Ob in den Abbildungen dargestellte Unterschiede signifikant sind oder nicht, wird unter den jeweiligen Abbildungen beschrieben.

In manchen Kapiteln stehen neben dem sozialen Wandel und den Gruppenunterschieden Zusammenhänge zwischen verschiedenen Indikatoren im Mittelpunkt (vgl. Kapitel 8, 9, 10, 18, 24). Ob entsprechende Zusammenhänge signifikant sind, wird (wie oben beschrieben) über multivariate Analyseverfahren geprüft. Gleichzeitig werden in diesen Kapiteln die in den Abbildungen berichteten Werte aus den Regressionen selbst berechnet. Auch in diesen Analysen wird für die Stratifizierungsvariablen kontrolliert, die allerdings an den Verteilungen des Mikrozensus zentriert wurden. Auf diese Weise können die berechneten Maße als gewichtet interpretiert werden. Zugleich sind sie um mögliche Einflüsse von Alter, Geschlecht und Landesteil bereinigt.

Welches statistische Verfahren zur Signifikanzprüfung und zur Darstellung der Ergebnisse zum Einsatz kommt, wird in den jeweiligen Kapiteln berichtet. Grundsätzlich wird bei allen Analysen ein Signifikanzniveau von fünf Prozent veranschlagt.

von Einwanderern bei Bevölkerungsbefragungen. Zeitschrift für Soziologie, 223-242.

Blom, A. G., \& Schröder, M. (2011). Sample composition 4 years on: Retention in Share Wave 3. In: M. Schröder (Hrsg.) Retrospective data collection in the Survey of Health, Ageing and Retirement in Europe (S. 55-61). Mannheim: Mannheim Research Institute for the Economics of Aging (MEA).

Börsch-Supan, A., Brandt, M., Hunkler, C., Kneip, T., Korbmacher, J., Malter, F., Schaan, B., Stuck, S., \& Zuber, S. (2013). Data resource profile: the Survey of Health, Ageing and Retirement in Europe (SHARE). International Journal of Epidemiology, 42(4), 9921001.

Blohm, M., \& Diehl, C. (2001). Wenn Migranten Migranten befragen: Zum Teilnahmeverhalten 
Brick, J. M., \& Williams, D. (2013). Explaining rising nonresponse rates in cross-sectional surveys. The ANNALS of the American Academy of Political and Social Science, 645(1), 36-59.

Brüderl, J., Schmiedeberg, C., Castiglioni, L., Arránz Becker, O., Buhr, P., Fuß, D., Ludwig, V., Schröder, J., \& Schumann, N. (2015). The German Family Panel: Study Design and Cumulated Field Report (Waves 1 to 6). O. O.: pairfam.

Deding, M., Fridberg, T., \& Jakobsen, V. (2008). Nonresponse in a survey among immigrants in Denmark. Survey Research Methods, 2(3), 107-121.

Engstler, H., \& Motel-Klingebiel, A. (2010). Datengrundlagen und Methoden des Deutschen Alterssurveys (DEAS). In: A. Motel-Klingebiel, S. Wurm \& C. Tesch-Römer (Hrsg.) Altern im Wandel. Befunde des Deutschen Alterssurveys (DEAS) (S. 34-60). Stuttgart: Kohlhammer.

Engstler, H., \& Wurm, S. (2006). Datengrundlagen und Methodik. In: C. Tesch-Römer, H. Engstler \& S. Wurm (Hrsg.) Altwerden in Deutschland. Sozialer Wandel und individuelle Entwicklung in der zweiten Lebenshälfte (S. 47-83). Wiesbaden: VS Verlag für Sozialwissenschaften.

Hoyer, W. J., Stawski, R. S., Wasylyshyn, C., \& Verhaeghen, P. (2004). Adult age and digit symbol substitution performance: a meta-analysis. Psychology and Aging, 19(1), 211-214.

Huisman, M., Poppelaars, J., Horst, M. van der, Beekman, A. T. F., Brug, J., Tilburg, T. G. van, \& Deeg, D. J. H. (2011). Cohort profile: the longitudinal aging study Amsterdam. International Journal of Epidemiology, 40(4), 868-876.

Klaus, D., \& Engstler, H. (2012). Daten und Methoden des Deutschen Alterssurveys (DEAS). In A. MotelKlingebiel, S. Wurm \& C. Tesch-Roemer (Hrsg.) Kontexte des Alterns. Längsschnittliche Befunde des DEAS 2011 (S. 13-27). Berlin: Deutsches Zentrum für Altersfragen.

Künemund, H. (2000). Datengrundlage und Methoden. In: M. Kohli \& H. Künemund (Hrsg.) Die zweite Lebenshälfte. Gesellschaftliche Lage und Partizipation im Spiegel des Alters-Survey (S. 33-40). Opladen: Leske + Budrich.

Laurie, H., \& Lynn, P. (2009). The use of respondent incentives on longitudinal surveys. In: P. Lynn (Hrsg.) Methodology of longitudinal surveys (S. 205-233). Chichester: Wiley.

Leeuw, E. D. de, \& Heer, W. de (2002). Trends in household survey nonresponse: A longitudinal and international comparison. In: M. R. Groves, D. A. Dillman, J. L. Eltinge \& R. J. A. Little (Hrsg.) Survey nonresponse (S. 41-54). New York: Wiley.
Michaud, P. C., Kapteyn, A., Smith, J. P., \& Soest, A. van (2011). Temporary and permanent unit non-response in follow-up interviews of the Health and Retirement Study. Longitudinal and Life Course Studies, 2(2), 145169.

Motel-Klingebiel, A., Klaus, D., \& Simonson, J. (2014). Befragungen von älteren und alten Menschen. In: N. Baur \& J. Blasius (Hrsg.) Handbuch Methoden der empirischen Sozialforschung (S. 781-786). Wiesbaden: Springer VS.

Nolan, A., O'Regan, C., Dooley, C., Wallace, D., Hever, A., Cronin, H., Hudson, E., \& Kenny, R. A. (Hrsg.). (2014). The over 50s in a changing ireland. Economic Circumstances, Health and Well-Being. Dublin: The Irish Longitudinal Study on Ageing (TILDA).

Pforr, K., Blohm, M., Blom, A. G., Erdel, B., Felderer, B., Fräßdorf, M., Hajek, K., Helmschrott, S., Kleinert, C., Koch, A., Krieger, U., Kroh, M., Martin, S., Saßenroth, D., Schmiedeberg, C., Trüdinger, E.-M., \& Rammstedt, B. (2015). Are incentive effects on response rates and nonresponse bias in large-scale, face-to-face surveys generalizable to Germany? Evidence from ten experiments. Public Opinion Quarterly, Online first (June), 1-29. doi: 10.1093/poq/nfv014.

Ryu, E., Couper, M. P., \& Marans, R. W. (2006). Survey incentives: Cash vs. in-kind; face-to-face vs. mail; response rate vs. nonresponse error. International Journal of Public Opinion Research, 18(1), 89-106.

Schiel, S., Dickmann, C., \& Aust, F. (2011). Methodenbericht Deutscher Alterssurvey (DEAS): 4. Befragungswelle. Panelbefragung 2011. Bonn: Infas Institut für angewandte Sozialwissenschaft $\mathrm{GmbH}$.

Schiel, S., Hess, D., \& Gilberg, R. (2003). Alterssurvey - Die zweite Lebenshälfte. Methodenbericht zur Erhebung der zweiten Welle 2002. Bonn: infas Institut für angewandte Sozialwissenschaft $\mathrm{GmbH}$.

Schiel, S., Kerr, P., Dickmann, C., \& Aust, F. (2015). Deutscher Alterssurvey (DEAS): Methodenbericht zur Durchführung der fünften Erhebungswelle 2014. Bonn: infas Institut für angewandte Sozialwissenschaft $\mathrm{GmbH}$.

Schiel, S., \& Smid, M. (2009). Alterssurvey 2008 - Die zweite Lebenshälfte. Durchführung der 3. Befragungswelle. Methodenbericht. Bonn: Infas Institut für angewandte Sozialforschung $\mathrm{GmbH}$.

Schnell, R. (1997). Nonresponse in Bevölkerungsumfragen. Opladen: Leske + Budrich.

Schröder, M. (2008). Attrition. In: A. Börsch-Supan, A. Brugiavini, H. Jürges, A. Kapteyn, J. Mackenbach, J. Siegrist \& G. Weber (Hrsg.) Health, Aging and Retirement in Europe (2004-2007) - Starting the longitutinal dimension (S. 327-332). Mannheim: 
Mannheim Research Institute for the Economics of Aging (MEA).

Smid, M., Hess, D., \& Gilberg, R. (1997). Alterssurvey - Lebensentwürfe, Einstellungen, Bedürfnislagen und Sinnstrukturen älterwerdender Menschen. Methodenbericht zur Erhebung der ersten Welle. Bonn: Infas Institut für angewandte Sozialforschung $\mathrm{GmbH}$.

Stadler, B. (2009). Die Befragung von MigrantInnen in Stichprobenerhebungen. In: M. Weichold, J. Bacher \& C. Wolf (Hrsg.) Umfrageforschung. Herausforderungen und Grenzen (S. 275-291). Wiesbaden: Springer VS.

Statistisches Bundesamt (2015b). Pflegestatistik 2013. Wiesbaden: Statistisches Bundesamt.

Statistisches Bundesamt (2015a). EU-SILC Nettoäquivalenzeinkommen 2013 nach Altersgruppen. Abruf aus der Genesis Online-Datenbank des Statistischen Bundesamts, Stand: 28.05.2015.

Statistisches Bundesamt (2015). Mikrozensus 2014 Sonderauswertungen für das Deutsche Zentrum für Altersfragen. Wiesbaden: Statistisches Bundesamt.

Statistisches Bundesamt (2014). Statistik der schwerbehinderten Menschen 2013. Wiesbaden: Statistisches Bundesamt.

Stoop, I., Billiet, J., Koch, A., \& Fitzgerald, R. (2010). Improving survey response: Lessons learned from the European Social Survey. Chichester: John Wiley \& Sons.
Tewes, U. (1994). Hamburg-Wechsler-Intelligenztest für Erwachsene, Revision 1991. Bern: Huber.

TNS infratest Sozialforschung (2013). SOEP 2012 Methodenbericht zum Befragungsjahr 2012 (Welle 29) des Sozio-oekonomischen Panels. Berlin: Deutsches Institut für Wirtschaftsforschung Berlin.

UNESCO (2006). International Standard Classification of Education ISCED 1997. Paris: UNESCO.

Vaz Fragoso, C. A., Gahbauer, E. A., Ness, P. H. van, Concato, J., \& Gill, T. M. (2008). Peak expiratory flow as a predictor of subsequent disability and death in community living older persons. Journal of the American Geriatrics Society, 56(6), 1014-1020.

Wasmer, M., Blohm, M., Walter, J., Scholz, E., \& Jutz, R. (2014). Konzeption und Durchführung der "Allgemeinen Bevölkerungsumfrage der Sozialwissenschaften" (ALLBUS) 2012. Mannheim. Online: https://dbk.gesis. org/dbksearch/file.asp?file=ZA4614_mb.pdf.

Weir, D. R., Faul, J. D., \& Langa, K. M. (2011). Proxy interviews and bias in cognition measures due to nonresponse in longitudinal studies: a comparison of HRS and ELSA. Longitudinal and Life Course Studies, 2(2), 170-184.

Open Access Dieses Kapitel wird unter der Creative Commons Namensnennung 2.5 International Lizenz (http://creativecommons.org/licenses/by/2.5/deed.de) veröffentlicht, welche die Nutzung, Vervielfältigung, Bearbeitung, Verbreitung und Wiedergabe in jeglichem Medium und Format erlaubt, sofern Sie den/die ursprünglichen Autor(en) und die Quelle ordnungsgemäß nennen, einen Link zur Creative Commons Lizenz beifügen und angeben, ob Änderungen vorgenommen wurden.

Die in diesem Kapitel enthaltenen Bilder und sonstiges Drittmaterial unterliegen ebenfalls der genannten Creative Commons Lizenz, sofern sich aus der Abbildungslegende nichts anderes ergibt. Sofern das betreffende Material nicht unter der genannten Creative Commons Lizenz steht und die betreffende Handlung nicht nach gesetzlichen Vorschriften erlaubt ist, ist für die oben aufgeführten Weiterverwendungen des Materials die Einwilligung des jeweiligen Rechteinhabers einzuholen. 